\title{
La colección "Gonzalo y Tarín" de fósiles históricos de la provincia de Granada del Museo Geominero (Instituto Geológico y Minero de España, Madrid)
}

\author{
S. Menéndez y M. Victoria Quiralte \\ Museo Geominero, Instituto Geológico y Minero de España, Ríos Rosas 23, 28003 Madrid. \\ s.menendez@igme.es; mv.quiralte@igme.es
}

\begin{abstract}
RESUMEN
El valor e importancia de las colecciones de fósiles, minerales y rocas que alberga el Museo Geominero se debe a su origen, estrechamente relacionado con la Comisión del Mapa Geológico de España, institución germen del actual Instituto Geológico y Minero de España, brindándoles un marcado carácter histórico. Joaquín Gonzalo y Tarín fue el encargado por la Comisión de realizar las investigaciones geológicas en la provincia de Granada. Para la realización de estos estudios geológicos se recolectaban numerosos ejemplares fósiles que eran identificados taxonómicamente y que luego pasaban a formar parte del grueso del material depositado en la litoteca de la Comisión, origen del futuro Museo que se inauguró en 1927. Sin embargo, a lo largo del tiempo la documentación correspondiente a estas colecciones ha sufrido cierto menoscabo, de manera que en muchas ocasiones es necesario llevar a cabo arduas labores de investigación para establecer su autoría. Precisamente son estas labores las que han propiciado la identificación de los fósiles recogidos por Gonzalo y Tarín en la provincia de Granada. Se trata de un conjunto de 84 ejemplares entre los 199 registros que actualmente conforman el total de la colección de fósiles de Granada depositados en el Museo Geominero. Se ha elaborado un catálogo con todos ellos, describiendo el etiquetado adherido y/o anexo que presentaba cada caso y tipificándose dos nuevos tipos de etiquetas adheridas correspondientes a la Comisión.
\end{abstract}

Palabras clave: Comisión del Mapa Geológico, Fósiles, Granada, Historia de la Geología, Instituto Geológico y Minero de España

\section{The "Gonzalo y Tarín" historical fossil collection of the province of Granada from the Geominero Museum (IGME, Madrid, Spain)}

\begin{abstract}
The value and importance of the collections of fossils, minerals and rocks housed in the Geominero Museum is due to their origin, closely related to the Commission for the Geological Map of Spain, the germ institution of the current Geological and Mining Institute of Spain. Joaquín Gonzalo y Tarín was commissioned to carry out geological research in the province of Granada. In order to carry out these geological studies, many fossils were collected and taxonomically identified. These specimens later became part of the bulk of the material deposited in the rock collection repository of the Commission for the Geological Map of Spain, the origin of the future Museum that was inaugurated in 1927. However, the documentation corresponding to these collections has suffered some damage over time, so that on many occasions it is necessary to carry out arduous research work to establish its authorship. It is precisely this work that has led to the identification of fossils collected by Gonzalo y Tarín in the province of Granada. It is a set of 84 specimens, among the 199 records that currently make up the total collection of the Granada fossils deposited in the Geominero Museum. A catalogue of them all has been prepared, describing the labelling stuck and/or attached presented by each record, typifying two new types of labels related to the Commission.
\end{abstract}

Keywords: Commission for the Geological Map of Spain, fossils, Geological Survey of Spain, Granada, History of Geology. 


\section{Introducción}

La importancia de las colecciones geológicas depositadas en el Museo Geominero viene dada por su notable valor histórico. Una buena parte de ellas fueron incorporadas durante la segunda mitad del siglo XIX y la primera mitad del XX, mientras ilustres ingenieros de minas destinados en la Comisión del Mapa Geológico de España o en los distritos mineros, llevaban a cabo los trabajos para la realización de la cartografía geológica nacional. Estos reunieron los datos y las colecciones de rocas, minerales y fósiles que dieron lugar a la elaboración de los mapas geológicos provinciales que servirían de base para la construcción del mapa geológico a escala nacional y a la primera síntesis de calado de la geología de España elaborada por Lucas Mallada. La Comisión del Mapa Geológico atravesó por diversas etapas a lo largo de su historia, que en algunas ocasiones conllevó el cambio de nombre. Desde su creación en 1849 bajo la denominación de Comisión para la Carta Geológica de Madrid y General del Reino (fundada por Isabel II mediante Real Decreto de 12 de julio del mismo año), pasó a denominarse, en etapas sucesivas, Comisión del Mapa Geológico de España o Instituto Geológico de España, hasta que llegó a su designación actual de Instituto Geológico y Minero de España (IGME) (Rábano, 2015). Las diferentes épocas atravesadas por la institución también comportaron diferentes traslados de sede, con los correspondientes desplazamientos y remodelación de sus fondos. Estos acontecimientos produjeron importantes pérdidas de ejemplares y de su información. Estas circunstancias, junto con la ausencia de un inventario original, han propiciado que en los últimos veinticinco años se vengan desarrollando investigaciones sistemáticas de las colecciones históricas con el objetivo de recuperar y poner en valor las mismas. Dichas labores de investigación de los fondos históricos tienen el propósito de identificary reintegrar las colecciones de autor derivadas de los trabajos pioneros realizados por los miembros de la Comisión en el siglo XIX y principios del XX. De este modo, las primeras investigaciones sobre las colecciones históricas del Museo Geominero llevadas a cabo con este propósito, se centraron en los fósiles recolectados por autores tan relevantes como Casiano de Prado (Arbizu et al., 1996; Rábano, 1998; Martínez y Rábano, 1999) o Lucas Mallada (Lozano et al., 1999; Rábano, 1999, 2000; Rábano y Gutiérrez-Marco, 1999) y la colección Schulz de rocas de Galicia (Lozano et al., 2005a). La identificación de los ejemplares históricos en estos casos se realizó, en primer término, cotejando las ilustraciones publicadas en distintas

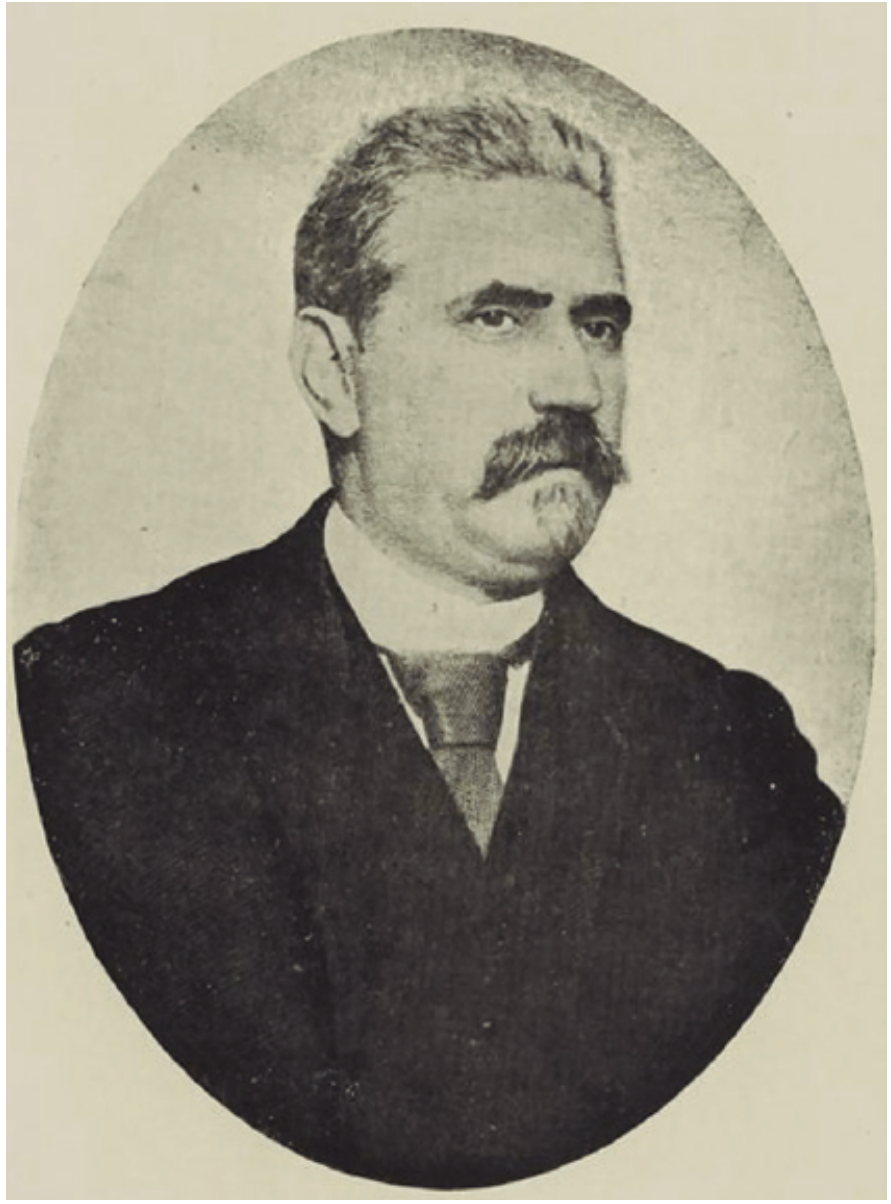

Figura 1. Retrato de Joaquín Gonzalo y Tarín (1838-1910) (tomado de Romero, 2013).

Figure 1. Portrait of Joaquín Gonzalo y Tarín (1838-1910) (from Romero, 2013).

épocas y los ejemplares del Museo. Además, la identificación de las etiquetas anexas y adheridas a cada espécimen, características de la Comisión del Mapa Geológico de España y su tipificación (ver siguientes referencias), también fue de vital importancia para el reconocimiento e identificación de los ejemplares originales de rocas y fósiles recolectados durante los trabajos llevados a cabo por los miembros de la Comisión. De esta manera se han podido reconocer los fósiles históricos de las provincias de Córdoba (Rábano, 1999), Jaén (Rábano, 2000), Aragón (Rábano y Delvene, 2003; De la Fuente et al., 2008), Barcelona (Lozano et al., 2005b), León (Rábano, 2006), Extremadura (Menéndez y Rábano, 2010) y Huelva (Menéndez et al., 2016). De la misma forma se ha procedido con las colecciones petrológicas, encontrándose actualmente diferenciadas las rocas históricas de las provincias de Barcelona (Lozano y Rábano, 2001), Zaragoza (Lozano y Rábano, 2004) y Huesca (González 
Laguna et al., 2007). Recientemente, y siguiendo los criterios utilizados en estos trabajos previos, se ha realizado una primera aproximación al catálogo de los ejemplares recolectados originalmente para la realización de la cartografía geológica provincial de Granada por Joaquín Gonzalo y Tarín en 1881. Este trabajo preliminar puede consultarse en Menéndez y Quiralte (2019).

\section{Joaquín Gonzalo y Tarín y la colección paleontológica de Granada en el Museo Geominero}

\section{Obra de Joaquín Gonzalo y Tarín (1838-1910)}

Los trabajos de la Comisión del Mapa Geológico para llevar a cabo la cartografía en la provincia de Granada correspondieron al ingeniero de minas Joaquín Gonzalo y Tarín (1838-1910) (Fig. 1). Tras finalizar sus estudios en la Escuela de Minas en 1865, al año siguiente, en 1866, se incorporó al distrito minero de Huelva. Allí desempeñó su labor hasta su incorporación definitiva en 1878 a la Comisión del Mapa Geológico, que por entonces se encontraba bajo la dirección del prolijo Manuel Fernández de Castro (1825-1895). Joaquín Gonzalo y Tarín fue vocal de la Comisión del Mapa Geológico hasta 1905, momento en el que fue jubilado del servicio, muriendo apenas unos pocos años después, en 1910 (Rábano, 2015; Suárez Suárez et al., 2017). La experiencia obtenida por Joaquín Gonzalo y Tarín durante su trabajo en el distrito minero de Huelva, así como en estudios geológicos previos publicados acerca de las provincias de Huelva (Gonzalo y Tarín, 1878, 1885, 1886-1887, 1888) y Badajoz (Gonzalo y Tarín, 1879), además del reconocimiento que había llevado a cabo de las provincias de Málaga, Sevilla y Almería (Gonzalo y Tarín, 1882a,b), le permitió ser la persona encargada de intentar dar respuesta al difícil problema que ofrecían en aquel momento los terrenos más antiguos de la Sierra Nevada en la provincia de Granada. Parte del trabajo que realizó Joaquín Gonzalo y Tarín al respecto, quedó recogido en la reseña publicada en el volumen correspondiente del Boletín de la Comisión del Mapa Geológico de España del año 1881. En el prólogo de este volumen se advierte que el autor tuvo que resumir considerablemente el texto de la memoria, para que pudiera formar parte del mismo, debido a la falta de espacio en este volumen. Fundamentalmente se acortaron las partes referentes a los aspectos físicos y de criaderos minerales, teniendo en cuenta que estas serían ampliadas debidamente una vez se publicara la Memoria definitiva, hecho que no ocurrió después, quedando esta reseña como memoria geológica de referencia para la provincia de Granada.

\section{La colección paleontológica de Granada en el Museo Geominero}

Actualmente las colecciones y fondos paleontológicos del Museo Geominero superan los 59.300 ejemplares, entre los que 199 proceden de la provincia de Granada (un $0,35 \%$ del total). Aunque el número de fósiles provenientes de esta provincia no es elevado, su relevancia es manifiesta. La mayoría de ellos están incluidos, hoy en día, en la colección más numerosa del museo, la de "Fósiles Invertebrados y Flora Españoles", que cuenta con algo más de 45.200 especímenes $(0,47 \%$ del total de esta colección corresponden a Granada). El conjunto de 199 fósiles granadinos, está constituido por los siguientes ejemplares: 69 de edad Jurásico; 1 de Cretácico; 8 de Paleógeno-Eoceno; 87 de Neógeno-Mioceno; 8 de Neógeno-Plioceno y 26 son cuaternarios. Muchos de ellos tienen su origen en la realización de la primera cartografía geológica publicada de la provincia de Granada (Gonzalo y Tarín, 1881) (Fig. 2). Este trabajo detalló, entre otras cosas, la presencia de determinadas especies fósiles en muchas de las zonas que se caracterizaron. Por tanto, los restos fósiles que se incorporaron a las colecciones del Museo Geominero (ver Fig. 3), procedían de las diversas áreas geológicas diferenciadas en el estudio cartográfico realizado y que fueron denominadas así originalmente: Época primaria 1) periodo estrato-cristalino (azoico); 2) sistema "Siluriano" -no encontrados restos paleontológicos-. Época secundaria 3) sistema Triásico -no encontrados restos paleontológicos-; 4) sistema Jurásico (28 taxones); 5) sistema "Cretáceo" (1 taxón). Época Terciaria 6) sistema Eoceno (8 taxones); 7) sistema Mioceno (23 taxones); 8) sistema Plioceno (5 taxones). Época "Posterciaria o cainozoica" 9) sistema Pleistoceno -no encontrados restos paleontológicos-. Todos estos ejemplares fósiles fueron determinados por Lucas Mallada (profesor de Paleontología de la Escuela de Ingenieros de Minas en ese momento). Desgraciadamente, como se verá más adelante, no todo este conjunto completo de fósiles se conserva actualmente en las colecciones del Museo Geominero, siendo destacada la ausencia del fósil cretácico citado en la memoria original.

\section{Material y métodos}

La metodología seguida para la realización de este trabajo ha sido la misma que se describe en las revisiones realizadas en los últimos años sobre otras colecciones históricas de rocas y fósiles del Museo Geominero (ver González Laguna et al., 2007; Lozano y Rábano, 2001; Lozano et al., 2005a,b; Menéndez y Rábano, 2010; Menéndez et al., 2016). El objeto de es- 


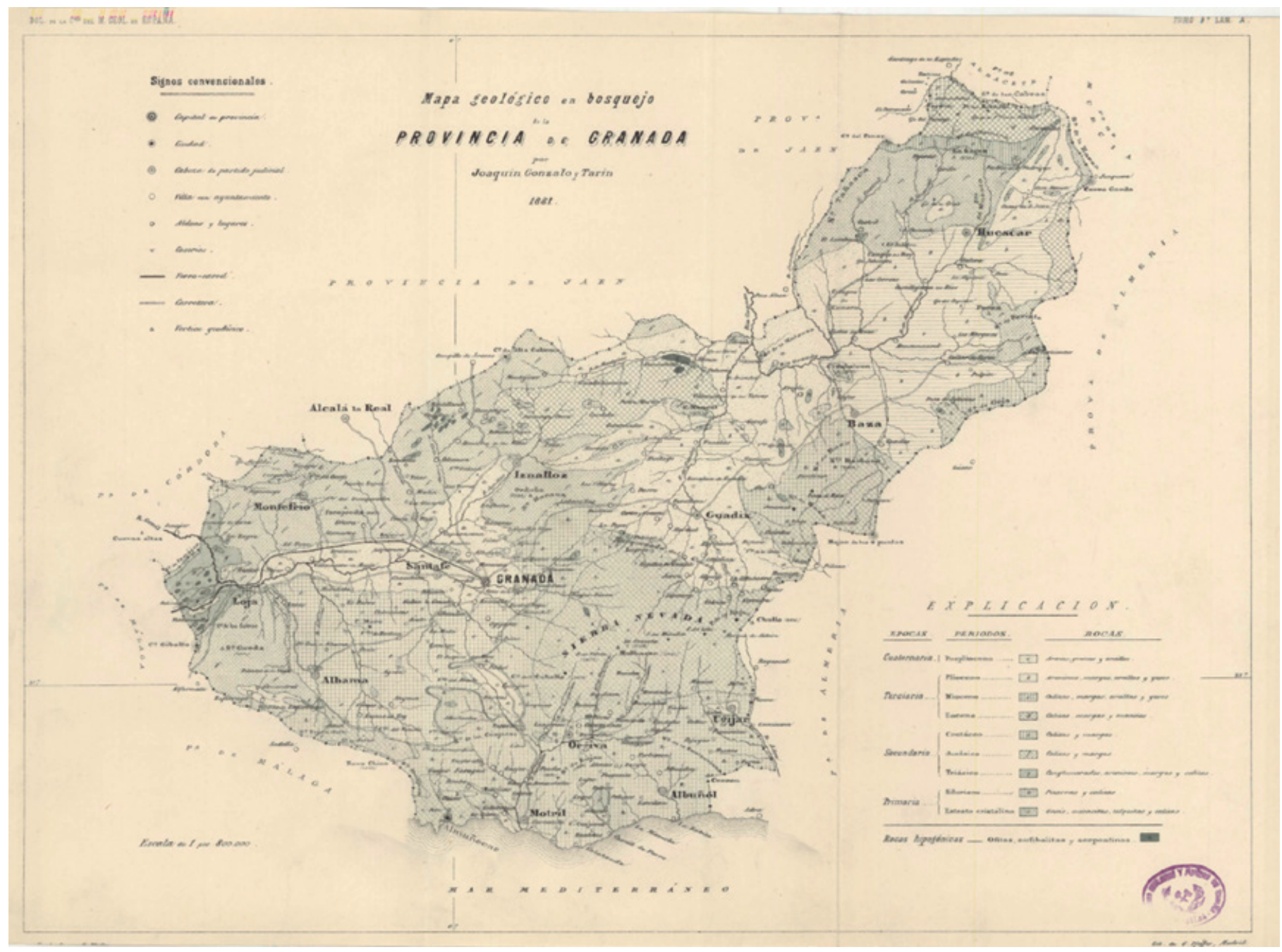

Figura 2. Mapa geológico de la provincia de Granada tomado del Boletín de la Comisión del Mapa Geológico de España,Tomo VIII, Provincia de Granada, de 1881 (Gonzalo y Tarín, 1881).

Figure 2. Geological map of the province of Granada taken from the Boletín de la Comisión del Mapa Geológico de España, Volume VIII, Province of Granada, 1881 (Gonzalo y Tarín, 1881).

tudio de esta revisión es la totalidad de los ejemplares fósiles procedentes de Granada depositados en el Museo Geominero (199 registros). Desgraciadamente, la mayor parte de la documentación original asociada a los trabajos de la Comisión del Mapa Geológico de España y la información sobre los ejemplares de Granada ingresados en el Museo Geominero como consecuencia de dichos trabajos, se ha perdido con el paso de los años. Por esta razón, se han examinado todos los ejemplares fósiles procedentes de Granada con el fin de localizar aquellos formatos de etiquetado, básicamente etiquetas adheridas y anexas, que pudieran corresponderse con los de la Comisión (Figs. 4 y 5). Entre el etiquetado anexo se han considerado las etiquetas originales de la Comisión y los borradores en papel previos a la realización de dichas etiquetas
(Lozano y Rábano, 2001). Igualmente se ha cotejado la información contenida en los registros de la base de datos del Museo Geominero con los listados de fósiles que aparecen en la reseña geológica sobre la provincia de Granada publicada por Gonzalo y Tarín (1881). El objetivo es identificar los ejemplares fósiles que podrían corresponderse con los que originalmente se recolectaron durante aquellos trabajos de la Comisión, comparando las coincidencias entre las edades y localidades/parajes en ambas fuentes (base de datos y bibliografía). Este procedimiento ha permitido identificar como históricos algunos ejemplares que carecían de etiquetado. Para la elaboración de este catálogo de fósiles históricos se han excluido aquellos en los que, aun siendo material antiguo, no ha podido establecerse una equivalencia entre sus localidades 


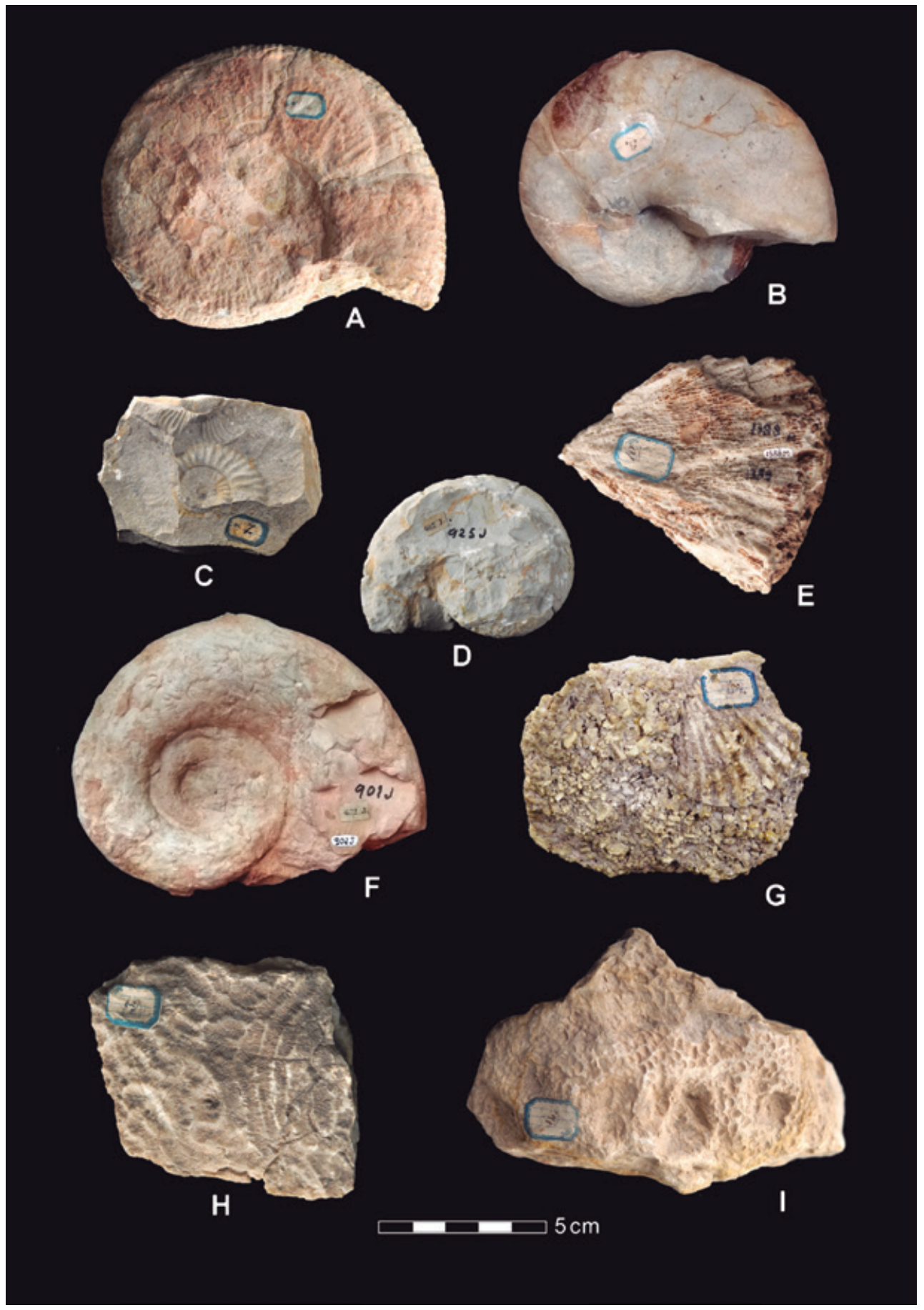

Figura 3. Ejemplares fósiles de la colección histórica de la provincia de Granada depositados en el Museo Geominero. A) MGM-1761J. Perisphinctes transitorius. Jurásico. El Manzanil-Loja. B) MGM-1039J. Holcophylloceras mediterraneum. Jurásico. EI Manzanil-Loja. C) MGM1676J. Bredyia uretae. Jurásico. Montillana. D) MGM-925J. Calliphylloceras calypso. Jurásico. El Manzanil-Loja. E) MGM-1388M. Prionastrea diversiformis. Mioceno. Alhama de Granada. F) MGM-902J. Lytoceras liebigi. Jurásico. El Manzanil-Loja. G) MGM-1631M. Aequipecten scabrella. Mioceno. Venta del Fraile, Padul. H) MGM-1760N. Scleractinia gen. indet. Eoceno. Puerto del Hornillo, Puebla de Don Fabrique. I) MGM-1384M. Heliastrea sp. Mioceno. Torre Cardela-Domingo Pérez. Los ejemplares A-C presentan etiqueta adherida "tipo 2". En los ejemplares D y F se observa una etiqueta adherida "tipo 17". Los ejemplares E, G-I muestran el nuevo tipo de etiqueta descrito como "tipo 24 ".

Figure 3. Fossil specimens from the historical collection of the province of Granada housed in the Geominero Museum. A) MGM-1761J. Perisphinctes transitorius. Jurásico. El Manzanil-Loja. B) MGM-1039J. Holcophylloceras mediterraneum. Jurásico. El Manzanil-Loja. C) MGM-1676J. Bredyia uretae. Jurásico. Montillana. D) MGM-925J. Calliphylloceras calypso. Jurásico. El Manzanil-Loja. E) MGM-1388M. Prionastrea diversiformis. Mioceno. Alhama de Granada. F) MGM-902J. Lytoceras liebigi. Jurásico. El Manzanil-Loja. G) MGM-1631M. Aequipecten scabrella. Mioceno. Venta del Fraile, Padul. H) MGM-1760N. Scleractinia gen. indet. Eoceno. Puerto del Hornillo, Puebla de Don Fabrique. I) MGM-1384M. Heliastrea sp. Mioceno. Torre Cardela-Domingo Pérez. Specimens A-C show a "type 2" stuck label. Specimens $D$ and $F$ have a "type 17" label. Specimens $E$ and G-I show the new described "type 24" label. 


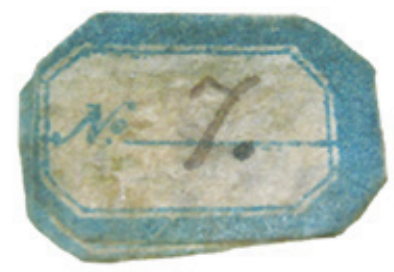

A

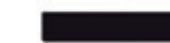

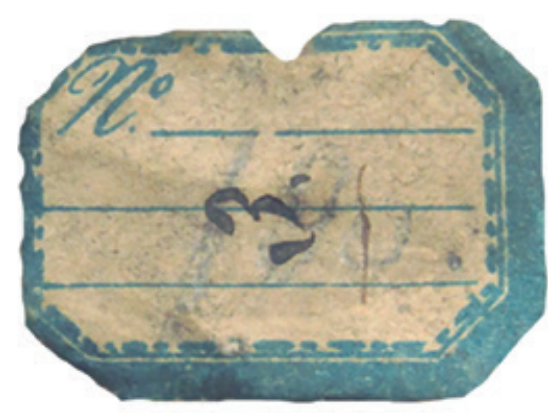

D

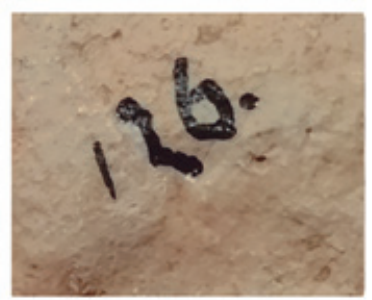

G

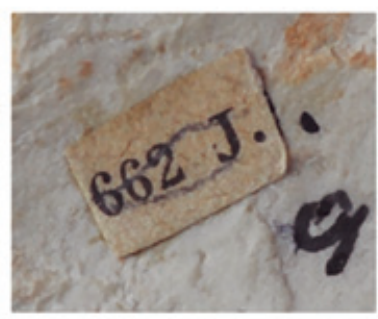

J

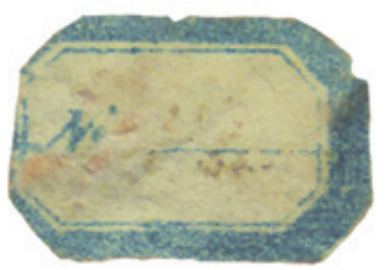

B

\section{$1 \mathrm{~cm}$}

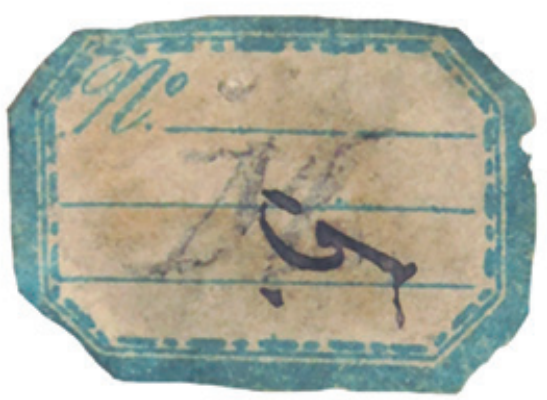

E

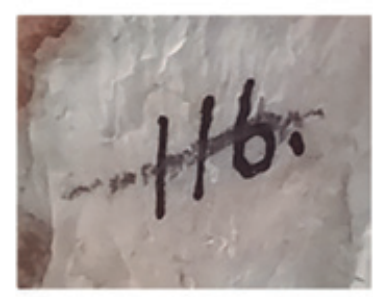

H

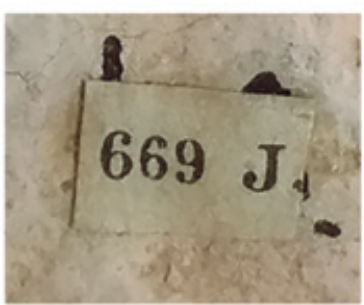

K

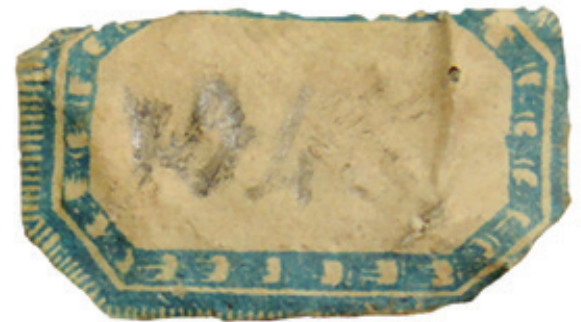

C

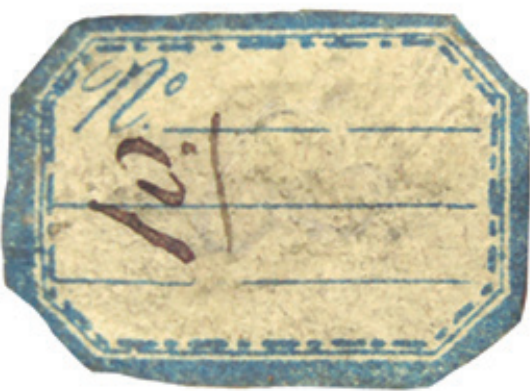

F

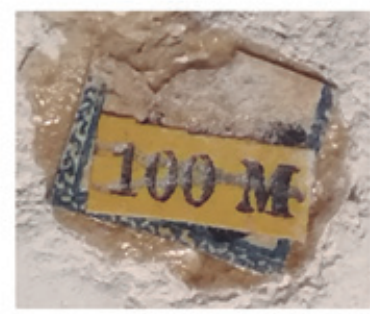

I

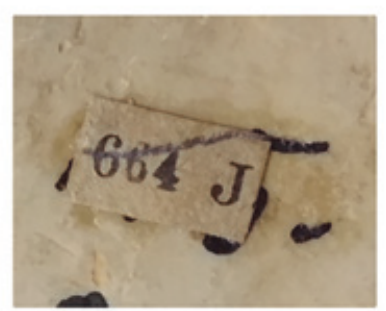

L

Figura 4. Etiquetas adheridas y otras numeraciones impresas con tinta en los ejemplares fósiles. A-B)Tipología 2 caracterizada por Lozano y Rábano (2001). C-F) Nuevas tipologías de etiquetas no reconocidas ni descritas previamente:Tipología 25 (C) y Tipología 24 (D-F). G-H) Números de sigla antiguos rotulados directamente sobre el ejemplar a plumilla; en el caso $\mathrm{H}$, este número aparece tachado. I) Dos etiquetas adheridas superpuestas: una etiqueta tipo 17 cubriendo parte de una etiqueta de tipo 16 . J-L) Etiquetas adheridas tipo 17 cubriendo siglas antiguas escritas directamente sobre el ejemplar a plumilla.

Figure 4. Stuck labels and other numbers marked directly on the fossil specimens. A-B) Typology 2 characterized by Lozano and Rábano (2001). C-F) New types of labels not previously recognized or described: Typology 25 (C) and Typology 24 (D-F). G-H) Old catalog numbers marked by nib directly on the specimen: in $\mathrm{H}$ this number is crossed out. I) Two superimposed stuck labels: a type 17 label covering part of a type 16 label. K-L) Stuck label type 17 covering old catalogue numbers marked by nib directly on the specimen. 


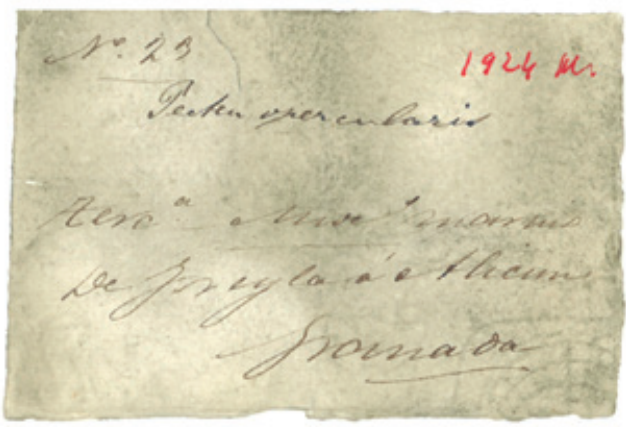

A

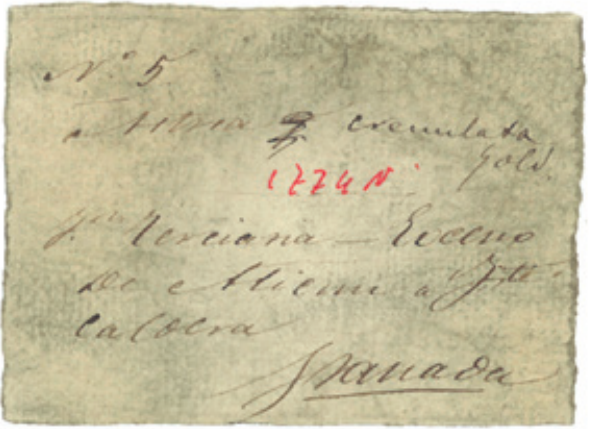

C

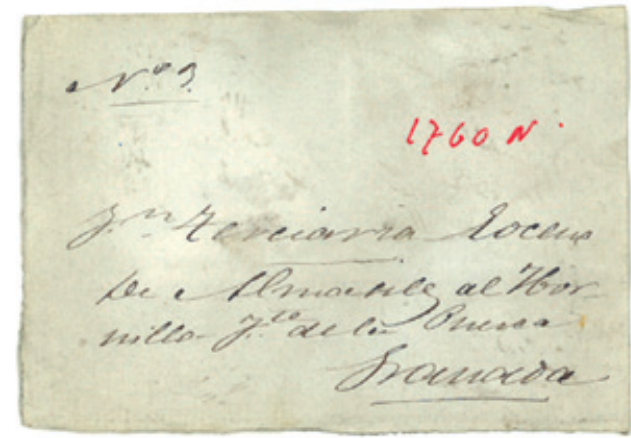

B

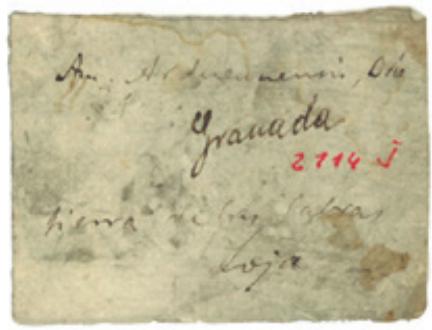

D

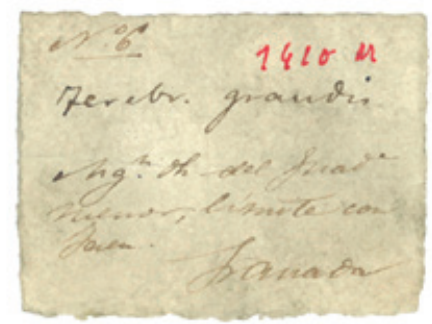

E

\section{$5 \mathrm{~cm}$}

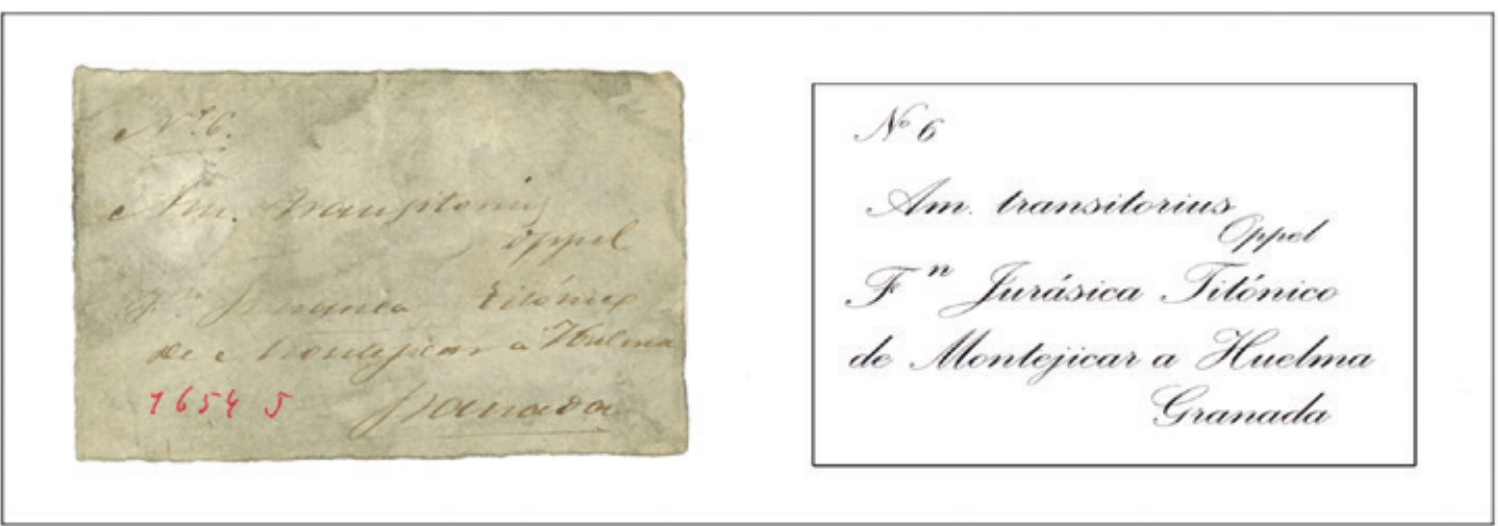

F

Figura 5. Diversos ejemplos de etiquetas de tipo "borrador" anexas a los ejemplares (A-E). Se trata de borradores previos a la realización de la etiqueta original de la Comisión del Mapa Geológico de España. En $\mathrm{F}$ se muestra la transcripción del texto original de la etiqueta $\mathrm{N}^{\circ} 6$ (MGM-1654J) para facilitar su lectura.

Figure 5. Several examples of "drafts" of the original labels attached to the fossils (A-E). These are previous drafts to the original label of the Commission for the Geological Map of Spain. F shows the transcription of the original text of the label $N^{\circ} 6$ (MGM-1654J) for easy reading.

de origen y/o carecían de etiquetado (bien etiquetas anexas, borradores o etiquetas adheridas).

Actualmente, debido a su fragilidad y valor histórico, las etiquetas anexas originales están archivadas en sobres de polipropileno químicamente neutro, de igual manera que otras etiquetas de colecciones históricas revisadas (Lozano y Rábano, 2001; Lozano et al., 2005b; González Laguna et al., 2007).

\section{Catálogo}

En la presentación del catálogo de la colección de los fósiles históricos de Granada se han ordenado los ejemplares por orden cronológico, de más antiguo a más moderno. En cada ejemplar catalogado se recopila la información existente sobre su numeración (aquellas siglas presentes en el espécimen, tanto ac- 
tuales como antiguas) clasificación taxonómica, localización geográfica, presencia y tipología de etiquetas (ya sean anexas y/o adheridas). La metodología seguida para recapitular, concentrar y codificar toda esta información sigue la descrita por Lozano et al., (2005b) con la adición de algunos códigos para recoger particularidades encontradas en la elaboración de este catálogo (ver tabla 1 con la codificación original añadiendo las modificaciones para este trabajo).

En el siguiente catálogo se recoge la nomenclatura taxonómica original reflejada en las etiquetas anexas de tipo borrador (Fig. 5), ya que los criterios para su elaboración han sido criterios históricos. No obstante, en la tabla 2 se detallan, a modo complementario, aquellos casos en los que sí se dispone actualmente de una taxonomía revisada. En el catálogo puede encontrarse también indicado entre paréntesis la correcta grafía de los topónimos que aparecen en el texto de la Memoria de Gonzalo y Tarín (1881) y que han sido reproducidos tal cual aparecen en el borrador anexo.

En cuanto a la numeración, los primeros números, subrayados, corresponden a la numeración que aparece en la esquina superior izquierda de la etiqueta anexa (ver Fig. 5). A continuación aparece en cursiva la numeración reflejada en las etiquetas adheridas a la roca (ver Figs. 4A-F, 4I-L). Al mismo tiempo, los diferentes tipos de etiquetas se han expresado como un superíndice detrás de los números en cursiva. Después, entre paréntesis se señalan los números impresos o rotulados directamente sobre el fósil (Figs. 4G-H), que a veces están cubiertos o tachados. En el examen de la colección hemos encontrado casos en los que, o bien las etiquetas adheridas tapaban otras etiquetas, superponiéndose varios tipos (Fig. 4I), o bien éstas cubrían siglas antiguas escritas directamente sobre el ejemplar a plumilla (Figs. 4J-L). Estas particularidades las hemos recogido independientemente y se reflejan en la tabla 1. Por último, los números en negrita indican la numeración del inventario actual del Museo Geominero.

\section{Jurásico}

- 7. $7^{2,2}$. 1676J. Ammonites variabilis. Jurásico. Montillana.

- 664 ${ }^{17}$.664J. Ammonites mediterraneum. Jurásico. EI Manzanil-Loja.

- 66417. [(DC)]. 929J. Ammonites mediterraneum. Jurásico. El Manzanil-Loja.

- 66417. 1041J. Ammonites mediterraneum. Jurásico.

El Manzanil-Loja.

- 32. 1039J. Ammonites mediterraneum. Jurásico. El Manzanil-Loja.

- 6. 1654J. Ammonites transitorius. Jurásico. De Mon- tejicar a Huelma.

- 927J. Ammonites transitorius. Jurásico. El Manzanil-Loja.

- 16. 17. $D C^{2}$. 1761J. "Ammonites transitorius". Jurásico. Sierra de las Cabras (borrador 16) y Cerro de las Monjas (borrador 17), Loja.

- 15. 2106J. Ammonites transitorius. Jurásico. Cerro de las Monjas, Loja.

- 66917. [(DC)]. 669J. Ammonites ptychoicus. Jurásico. El Manzanil-Loja.

- 66917. [(DC)]. 748J. Ammonites ptychoicus. Jurásico. El Manzanil-Loja.

- 980J. Ammonites ptychoicus. Jurásico. El Manzanil-Loja.

- 14. 2102J. Ammonites silesiacus. Jurásico. Cerro de las Monjas, Loja.

- 675 17 [(DC)]. 675J. Ammonites municipale. Jurásico. El Manzanil-Loja.

- 1053J. Ammonites municipale. Jurásico. El Manzanil-Loja.

- 67517. [(DC)]. 1055J. Ammonites municipale. Jurásico. El Manzanil-Loja.

- 1222J. Ammonites arduennensis. Jurásico. Cerro de las Monjas, Loja.

- SN. 2114J. Ammonites arduennensis. Jurásico. Sierra de las Cabras, Loja.

- 672 $17.672 \mathrm{~J}$. Ammonites liebigr. Jurásico. El Manzanil-Loja.

- 67217. 902J. Ammonites liebigi?. Jurásico. El Manzanil-Loja.

- (113). 905J. Ammonites liebigi?. Jurásico. El Manzanil-Loja.

- 1050J. Ammonites liebigi?. Jurásico. El Manzanil-Loja.

- 102. 10J. Ammonites bakeriae. Jurásico. Montillana.

- 58. $D C^{2}$. 982J. Ammonites arolicus. Jurásico. Sierra de las Cabras, Loja.

- 66117. (126). 1035J. Ammonites sp. Jurásico. El Manzanil-Loja.

- 1154J. Ammonites sp. Jurásico. El Manzanil-Loja.

- 66117. (126). 661J. Ammonites sp. Jurásico. El Manzanil-Loja.

- 66117. (127). 911J. Ammonites sp. Jurásico. El Manzanil-Loja.

- 66117. (126). 921J. Ammonites sp. Jurásico. El Manzanil-Loja.

- 662 ${ }^{17}$. [(DC)]. 925J. Ammonites sp. Jurásico. El Manzanil-Loja.

- 682 ${ }^{17}$. (116). 682J. Ammonites elimatus. Jurásico. El Manzanil-Loja.

- 1100J. Apthycus latus. Jurásico. Castril.

- 5817. (142). (12). 58J. Apthycus punctatus. Jurásico. Campotéjar.

- 6171. (142). 61J. Apthycus sparila. Jurásico. Campotéjar. 
Código o tipo de carácter

Subrayado
Cursiva
Negrita
Superindice Numérico
SN
DC
Parénteisis ()
Corchetes []

Descripción

Número presente en las etiquetas anexas

Número presente en las etiquetas adheridas

Número de inventario actual del museo

Tipo de etiqueta adherida reconocible $(2,16,17,24,25)$

Etiqueta adherida de tipo no reconocible $(0)$

Sin número. La etiqueta anexa (subrayado) o adherida (cursiva) no tiene número

Número desconocido. No se observa el número, porque la etiqueta está deteriorada o cubierto parcialmente por otra etiqueta

Número escrito directamente sobre el fósil (distinto de la sigla del inventario actual del museo)

Indica que el número escrito sobre el fósil está cubierto o tapado por una etiqueta

Las etiquetas adheridas sucesivas una encima de otra se separan por una coma

Tabla 1. Relación de los códigos y su descripción correspondiente utilizados para resumir la información de cada ejemplar, incluyendo la que aporta el etiquetado anexo y adherido. Modificado de Lozano et al., (2005b).

Table 1. List of the codes and their corresponding description used to summarize the information of each specimen, including that provided by the attached and stuck labelling. Modified from Lozano et al., (2005b).

\section{Eoceno}

- 5. 5 $5^{24}$ 1774N. Astrea crenulata. Eoceno. De Alicún (Alicún de Ortega) a Fuente Caldera.

- 1. 1763N. Heliastrea defrancei. Eoceno. De Almaciles a Santiago de la Espada (Hornillo), Granada.

- 3. $3^{0,24} \cdot 1760 \mathrm{~N}$. Scleractinia género indeterminado. Eoceno. De Almaciles al Hornillo- Fuente de la Puerca.

\section{Mioceno}

- 16. 1603M. Cardium sp. Mioceno. Alhama (Alhama de Granada).

- 6. 1410M. Terebratula grandis. Mioceno. Margen derecha del Guadiana-Menor, límite con Jaén.

- 6. 5053M. Terebratula grandis. Mioceno. Poblado del Negratín-Freila.

- 6. 5054M. Terebratula grandis. Mioceno. Poblado del Negratín-Freila.

- 2. $2^{24}$. (DC). 1631M. Pecten opercularis. Mioceno. ViIlares -Venta del Fraile, Padul, Granada.

- 1990M. Pecten opercularis. Mioceno. Padul.
- 5101M. Pecten opercularis. Mioceno. Padul.

- 5102M. Pecten opercularis. Mioceno. Padul.

- 5. 1688M. Pecten opercularis. Mioceno. Los Villares, Padul.

- 5. 5035M. Pecten opercularis. Mioceno. Los Villares, Padul.

- 5. 5036M. Pecten opercularis. Mioceno. Los Villares, Padul.

- 23. 1924M. Pecten opercularis. Mioceno. De Freyla

(Freila) a Alicún (Alicún de Ortega), Granada.

- 23. 5000M. Pecten opercularis. Mioceno. Freila.

- 23. 5001M. Pecten opercularis. Mioceno. Freila.

- 3. $3^{24} .1927 \mathrm{M}$. Pecten opercularis. Ostrea crassisima.

Mioceno. "Fragmento de texto no descrifrado" de Granada y Jaen. Cuesta de la Mala Mujer.

- 104 (escrito encima de un 94). 10425.1672M. Pecten sp.

Mioceno. Suerte Hundida, término de Albuñuelas.

- 104 (escrito encima de un 94). 4974M. Pecten sp. Mioceno. Suerte Hundida, Albuñuelas.

- 10. $10^{24}$. 1388M. Prionastrea diversiformis. Mioceno. 12 kilómetros de Alhama (Alhama de Granada) camino a Zafarraya, Granada.

- 14. 1424.1384M. Heliastrea sp. Mioceno. Cardela (Torre Cardela). 


\begin{tabular}{|c|c|c|c|c|c|c|c|}
\hline \multirow{2}{*}{ Género } & \multirow{2}{*}{ especie } & \multirow{2}{*}{$\begin{array}{c}\text { Gonzalo y } \\
\text { Tarin, } 1881 \\
\text { Procedencia }\end{array}$} & \multicolumn{5}{|c|}{ Museo Geominero } \\
\hline & & & Procedencia & $\begin{array}{l}\text { Ejemplar } \\
\text { MGM }\end{array}$ & $\begin{array}{l}\text { Etiqueta } \\
\text { adherida }\end{array}$ & $\begin{array}{l}\text { Etiqueta } \\
\text { anexa }\end{array}$ & $\begin{array}{l}\text { Revisado } \\
\text { taxonomia }\end{array}$ \\
\hline \multicolumn{8}{|c|}{ JURÁSICO } \\
\hline \multirow{17}{*}{ Ammonites } & vanabilis? & 5,8 & 5 & $1676 \mathrm{~J}$ & $x$ & $x$ & Bredyia uretae \\
\hline & \multirow{2}{*}{ mediterraneum } & \multirow{2}{*}{1} & \multirow{2}{*}{1} & $\begin{array}{l}664 \mathrm{~J}, 929 \mathrm{~J}, \\
1041 \mathrm{~J}\end{array}$ & $\cdot$ & $\cdot$ & \multirow{2}{*}{$\begin{array}{l}\text { Holcophylloceras } \\
\text { mediterraneum }\end{array}$} \\
\hline & & & & $1039 \mathrm{~J}$ & $x$ & . & \\
\hline & \multirow{4}{*}{ transitonius } & \multirow{4}{*}{1,3} & 22 & $1654 \mathrm{~J}^{*}$ & - & $x$ & \multirow{3}{*}{$\begin{array}{l}\text { Penisphinctes } \\
\text { transitorius }\end{array}$} \\
\hline & & & \multirow{3}{*}{1} & $927 \mathrm{~J}$ & $\cdot$ & $\cdot$ & \\
\hline & & & & $1761 \mathrm{~J}$ & $x$ & $x$ & \\
\hline & & & & $2106 \mathrm{~J}$ & $\cdot$ & $x$ & $\begin{array}{l}\text { Paraulacosphinctes } \\
\text { sp. }\end{array}$ \\
\hline & ptychoicus* & 2 & 1 & $\begin{array}{l}669 \mathrm{~J}, 748 \mathrm{~J}, \\
980 \mathrm{~J}\end{array}$ & - & - & $\begin{array}{l}\text { Ptychophylloceras } \\
\text { ptychoicum }\end{array}$ \\
\hline & silesiacus & 1,2 & 2 & $2102 \mathrm{~J}$ & $\cdot$ & $x$ & Phylloceras sp. \\
\hline & municipale & 1 & 1 & $\begin{array}{l}675 \mathrm{~J}, 1053 \mathrm{~J}, \\
1055 \mathrm{~J}\end{array}$ & $\cdot$ & $\cdot$ & $\begin{array}{l}\text { Pterolytoceras } \\
\text { municipale }\end{array}$ \\
\hline & \multirow{2}{*}{ arduennensis* } & \multirow{2}{*}{2} & 1 & $1222 \mathrm{~J}$ & $\cdot$ & - & $\begin{array}{l}\text { Parawedekindia } \\
\text { arduennensis }\end{array}$ \\
\hline & & & 2 & $2114 \mathrm{~J}$ & - & $x$ & $\begin{array}{l}\text { Protacanthodiscus } \\
\text { sp. }\end{array}$ \\
\hline & liebigi?" & 4 & 1 & $\begin{array}{l}672 \mathrm{~J}, 902 \mathrm{~J} \\
905 \mathrm{~J}, 1050 \mathrm{~J}\end{array}$ & - & - & Lytoceras liebigi \\
\hline & bakeniae* & 9 & 5 & $10 \mathrm{~J}$ & $x$ & $\cdot$ & Decipia? bakeniae \\
\hline & arolicus & 2 & 2 & $982 \mathrm{~J}$ & $x$ & $x$ & $\begin{array}{l}\text { Trimarginites } \\
\text { arolicus }\end{array}$ \\
\hline & sin determinar & 1 & 1 & $\begin{array}{l}1035 \mathrm{~J}, 1154 \mathrm{~J}, \\
661 \mathrm{~J}, 911 \mathrm{~J}, \\
921 \mathrm{~J}, 925 \mathrm{~J} .\end{array}$ & $\cdot$ & $\cdot$ & $\begin{array}{l}\text { Calliphylloceras } \\
\text { calypso }\end{array}$ \\
\hline & elimatus & 1 & 1 & $682 \mathrm{~J}$ & - & - & $\begin{array}{l}\text { Haploceras } \\
\text { elimatum }\end{array}$ \\
\hline \multirow{3}{*}{ Apthycus } & latus & 9 & 9 & $1100 \mathrm{~J}$ & - & - & Laevaptychus latus \\
\hline & punctatus & 3,7 & 7 & $58 \mathrm{~J}$ & $\cdot$ & . & $\begin{array}{l}\text { Punctaptychus } \\
\text { punctatus }\end{array}$ \\
\hline & spanila & 6 & 6 & $61 \mathrm{~J}$ & - & - & $\begin{array}{l}\text { Lamellaptychus } \\
\text { sparsilamellosus }\end{array}$ \\
\hline \multicolumn{8}{|c|}{ EOCENO } \\
\hline Astrea & crenulata & 11 & 11 & $1774 \mathrm{~N}$ & $\mathrm{x}$ & $\mathrm{x}$ & - \\
\hline Heliastrea & defrancei & $\cdot$ & 10 & $1763 \mathrm{~N}$ & - & $x$ & $\cdot$ \\
\hline Meandrina & & 10 & 10 & $1760 \mathrm{~N}$ & $x$ & $x$ & Rugosa gen. indet. \\
\hline \multicolumn{8}{|c|}{ MIOCENO } \\
\hline Cardium $*$ & & 18 & 18 & $1603 \mathrm{M}$ & . & $x$ & $\begin{array}{l}\text { Aequipecten } \\
\text { scabrella }\end{array}$ \\
\hline Terebratula & grandis & 13 & 13 & $\begin{array}{l}1410 \mathrm{M}, 5053 \mathrm{M} \\
5054 \mathrm{M}\end{array}$ & $\cdot$ & $x$ & - \\
\hline \multirow{6}{*}{ Pecten } & \multirow{5}{*}{ opercularis } & \multirow{5}{*}{$\begin{array}{c}12,13,15,16 . \\
2023,24\end{array}$} & & $1631 \mathrm{M}$ & $x$ & $x$ & \multirow{2}{*}{$\begin{array}{l}\text { Aequipecten } \\
\text { scabrella }\end{array}$} \\
\hline & & & 20 & $\begin{array}{l}1990 \mathrm{M}, 5101 \mathrm{M} \\
5102 \mathrm{M}\end{array}$ & $\cdot$ & - & \\
\hline & & & & $\begin{array}{l}1688 \mathrm{M}, 5035 \mathrm{M}, \\
5036 \mathrm{M}\end{array}$ & - & $x$ & Aequipecten sp. \\
\hline & & & 13 & $\begin{array}{l}1924 \mathrm{M}, 5000 \mathrm{M}, \\
5001 \mathrm{M}\end{array}$ & $\cdot$ & $x$ & $\begin{array}{l}\text { Aequipecten } \\
\text { scabrella }\end{array}$ \\
\hline & & & 23 & $1927 M$ & $x$ & $x$ & Aequipecten sp. \\
\hline & & $\begin{array}{l}15,18,23,24 \\
25,28,29,30\end{array}$ & 24 & $1672 \mathrm{M}, 4974 \mathrm{M}$ & $x$ & $x$ & Aequipecten sp. \\
\hline Prionastrea* & diversiformis & 21 & 18 & $1388 \mathrm{M}$ & $x$ & $x$ & - \\
\hline Solenastrea* & turonensis & 21 & 14 & $1384 \mathrm{M}$ & $x$ & $x$ & Heliastrea sp.- \\
\hline Hydrobia & etrusca & $\cdot$ & 19 & $1203 \mathrm{M}$ & $x$ & $\cdot$ & - \\
\hline & & & & $114 \mathrm{M}$ & $x$ & $\cdot$ & $\cdot$ \\
\hline Helix & arbustorum & 14,17 & 17 & $\begin{array}{l}1297 \mathrm{M}, 5126 \mathrm{M}- \\
5145 \mathrm{M}\end{array}$ & - & $x$ & - \\
\hline & costata & 18 & & & & & \\
\hline Melanopsis & & 18 & 18 & 99M, 3895M & - & - & Melanopsis dufoun \\
\hline & & & PLIOC & NO & & & \\
\hline Cardium & edule & 26,27 & 26.27 & $\begin{array}{l}4611 \mathrm{M}, 672 \mathrm{M}, \\
676 \mathrm{M}\end{array}$ & $\cdot$ & $\cdot$ & Cerastoderma edule \\
\hline
\end{tabular}


- 10016,17. 1203M. Hydrobia etrusca. Mioceno. Arenas del Rey.

- SN $N^{0,17}$. 114M. Helix arbustorum. Mioceno. Loja.

- 5. 1297M. Helix arbustorum. Mioceno. Manantial del Cofín, Loja.

- 5. 5126M-5145M. Helix arbustorum. Mioceno. Manantial del Cofín, Loja.

- 9917. 99M. Melanopsis costata. Mioceno. Alhama de Granada.

- 9917. 3895M. Melanopsis costata. Mioceno. Alhama de Granada.

\section{Plioceno}

- 13217. [(DC)]. 4611M. Cardium edule. Mioceno. Caniles.

- 13217. 672M. Cardium edule. Mioceno. Caniles.

- 133 ${ }^{17}$.676M. Cardium edule. Mioceno. Benamaurel.

\section{Discusión}

Para la provincia de Granada se ha reconocido la presencia de 84 fósiles que han sido incluidos dentro del catálogo de fósiles históricos, y que fueron recogidos durante los trabajos de elaboración de la cartografía geológica provincial de Granada llevados a cabo en el siglo XIX (Gonzalo y Tarín, 1881). La distribución por edades es la siguiente: 34 registros del Jurásico, 3 del Eoceno, 44 del Mioceno y 3 del Plioceno (ver tabla 2 y Fig. 3). En algunos casos (señalados con un asterisco en la tabla 2) las localidades de procedencia de los ejemplares citados en la memoria de la cartografía y los depositados en el museo no coinciden, pero en el texto de la memoria se especifica la presencia de caliza fosilífera con numerosas especies fósiles sin deter- minar en esas localidades. El material en dichas localidades fue objeto de recolección, ya que todos ellos están acompañados de etiquetas adherida y/o anexa, signo inequívoco de su origen (Gonzalo y Tarín, 1881). En el caso de MGM-1603M (doble asterisco en la tabla 2) puede verse que en la etiqueta anexa (borrador) no aparece una identificación taxonómica antigua, sino moderna, escrita en rojo, y que pone Cardium sp. Con posterioridad, esta asignación taxonómica sufrió una revisión a su vez, con lo que actualmente se clasifica como Aequipecten scrabella. En la Memoria de 1881 (pág. 91) aparece una referencia a la aparición de restos variados de moluscos en Alhama de Granada, algunos sin identificar. Pensamos que este ejemplar se encontraba entre ellos.

Con respecto al etiquetado que presentaban estos fósiles (ver Figs. 3, 4 y 5), hemos podido constatar la presencia de cinco tipos distintos de etiquetas adheridas directamente a los ejemplares. Tres de las tipologías coinciden con las previamente caracterizadas como "tipo 2" (Figs. 3A-C; Figs. 4A-B), "tipo 16" (Fig. 4I) y "tipo 17" (Figs. 3D, 3F; Figs. 4I-L) en los trabajos previos realizados sobre la colección histórica de rocas y fósiles de Barcelona del Museo Geominero (Lozano y Rábano, 2001; Lozano et al., 2005b). Los otros dos tipos de etiquetas adheridas que hemos encontrado no habían sido descritos con anterioridad: una de ellas es la más numerosa (Figs. 3E, 3G-l; Figs. 4DF) y la otra está representada sólo en un caso (MGM1672M; Fig. 4C). Estas etiquetas han sido designadas como tipos " 24 " y " 25 " respectivamente y se describen a continuación:

-Tipo "24": Son etiquetas octogonales, con los lados inferior y superior más largos que el resto de los lados. La decoración es de color azul oscuro. Tienen un reborde externo ancho, seguido de una línea fina

4 Tabla 2. Listado de ejemplares fósiles recolectados por Gonzalo y Tarín (1881) con las determinaciones taxonómicas tal y como aparecen

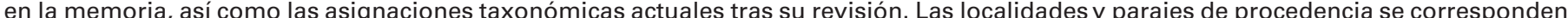
con los siguientes: (1) Manantial El Manzanil, Cerro de las Monjas (3 km SE de Loja); (2) Sierra de las Cabras y Gorda de Santa Lucía (S de

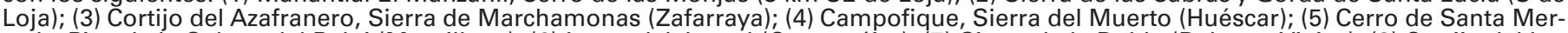

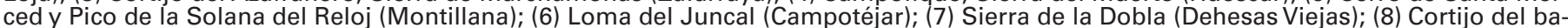

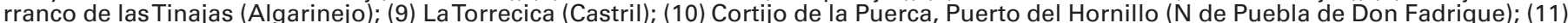

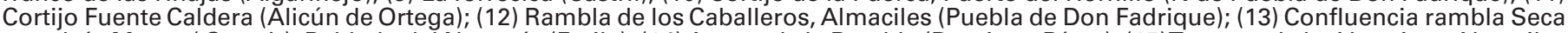

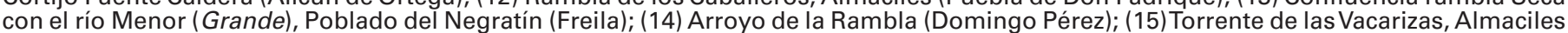

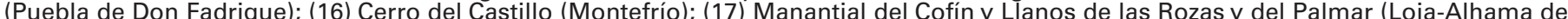

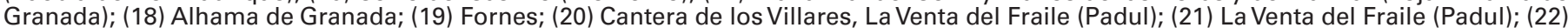

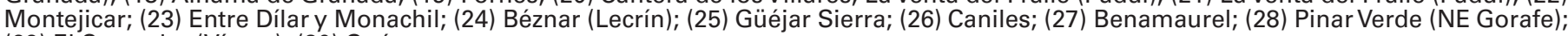
(29) El Caracolar (Víznar); (30) Quéntar.

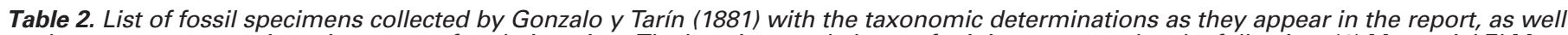

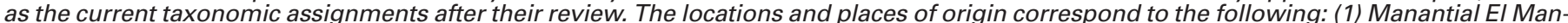

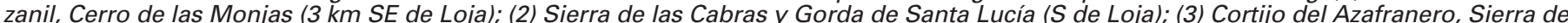

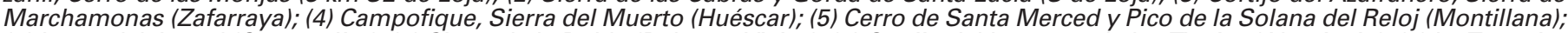

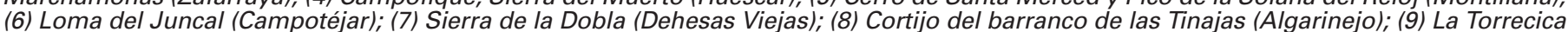

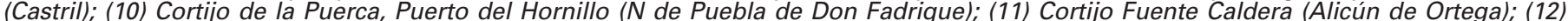

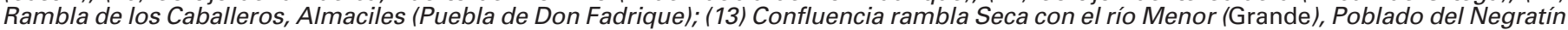

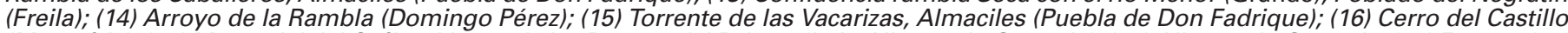

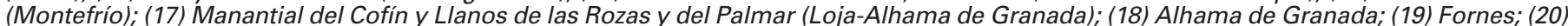

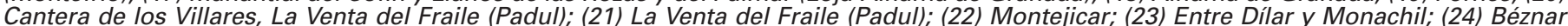
(Lecrín); (25) Güéjar Sierra; (26) Caniles; (27) Benamaurel; (28) Pinar Verde (NE Gorafe); (29) El Caracolar (Víznar); (30) Quéntar. 
y una tercera fila decorativa a modo de línea discontinua. En la parte central hay tres líneas paralelas y en la esquina superior aparece el encabezamiento " $N$ ". La presentan 6 ejemplares, casi la mitad de los que tienen etiquetas adheridas.

-Tipo "25": Sólo se ha encontrado en un ejemplar. Se trata de una etiqueta con la misma morfología que la anterior, es decir, octogonal alargada, pero la decoración, también azul oscuro, es en este caso más compleja. Desde el exterior hacia el interior se distinguen un reborde externo radiado, una franja azul lisa, una línea blanca y otra franja con decoración bandeada azul y blanco. El interior de esta etiqueta es liso.

Por otro lado, no ha sido encontrada ninguna etiqueta anexa original que se correspondiera directamente con las de la Comisión del Mapa Geológico, sino que todo el etiquetado se relaciona con borradores previos a la redacción de dichas etiquetas (Fig. $5 A-E)$. Sólo en diez casos los ejemplares presentaban doble etiquetado; es decir, la etiqueta adherida sobre el fósil directamente, y la etiqueta anexa que, como ya hemos comentado, se trata de borradores. En 14 ejemplares, sin embargo, sólo encontramos un tipo de etiquetado, siendo más numerosas las etiquetas anexas (en 10 casos) que las adheridas (4 ejemplares) (Tabla 2). Por último, hay que comentar el resto de los casos, que carecen de etiquetado, y que son los más numerosos. La asignación de este material a la categoría de "fósiles históricos" ha sido posible después de la detallada revisión de sus localidades de procedencia en la base de datos del Museo Geominero y su comparación con la información recogida en la bibliografía consultada. En la mayoría de las ocasiones la similitud entre la denominación de los parajes reflejada en ambas fuentes avala esta identificación.

\section{Conclusiones}

La ausencia de un archivo histórico en el Museo Geominero que recoja toda la información adicional de cada uno de los elementos geológicos que constituyen sus colecciones, es un inconveniente que se está tratando de superar con el desarrollo de una línea de investigación sobre las colecciones históricas que ha originado numerosos trabajos durante los últimos veinticinco años y que está permitiendo conocer el valor de la colección histórica de esta institución. Por tanto, la elaboración de este catálogo es reseñable, ya que caracteriza y pone en valor una nueva colección histórica, en este caso la colección de fósiles de Granada, que está constituida por 84 ejemplares. Para ello se ha seguido la metodología desarrollada previamente para la catalogación de otras coleccio- nes históricas de rocas y fósiles conservadas en el Museo Geominero. Esta metodología se basa en la identificación, caracterización y tipificación del etiquetado original y en el cotejo de la información presente en las mismas con los datos disponibles en las publicaciones originales y los registros de la base de datos del Museo Geominero. De este modo, se han reconocido etiquetas adheridas y anexas tipificadas previamente como pertenecientes a la Comisión del Mapa Geológico y se han definido dos nuevos tipos de etiquetas adheridas. El formato y las relaciones consignadas entre las diferentes etiquetas halladas en los fósiles de Granada permiten ampliar el estado de conocimiento de la historia de la propia Comisión, así como de la elaboración y autoría de la colección provincial de Granada.

\section{Agradecimientos}

Las autoras agradecen a $\mathrm{M}^{\mathrm{a}}$ José Torres la realización de las fotografías de los fósiles y etiquetas que aparecen en las figuras correspondientes. A Rodolfo Gozalo y otro revisor anónimo sus sugerencias y consejos que han mejorado el resultado final de este trabajo.

\section{Referencias}

Arbizu, M., Rábano, I. yTruyols, J. 1996. Trilobites del Museo Geominero. II. Las colecciones antiguas del Devónico de la Cordillera Cantábrica (N España). Boletín Geológico y Minero, 107 (1), 3-13.

De la Fuente, M., Rodrigo, A., Menéndez, S. y Lozano, R.P. 2008. Colecciones históricas en el Museo Geominero (IGME): la colección Donayre de fósiles de la provincia de Zaragoza. Libro de Resúmenes, XXIV Jornadas de la Sociedad Española de Paleontología, 99.

González Laguna, R., Lozano, R.P., Menéndez, S. y Abad, S. 2007. La colección histórica de rocas de la provincia de Huesca conservada en el Museo Geominero (IGME, Madrid): catalogación e interpretación histórica. Boletín Geológico y Minero, 118 (1), 127-140.

Gonzalo y Tarín, J. 1878. Reseña geológica de la provincia de Huelva. Boletín de la Comisión del Mapa Geológico de España, 5, 1-138.

Gonzalo y Tarín, J. 1879. Reseña físico-geológica de la provincia de Badajoz. Boletín de la Comisión del Mapa Geológico de España, 6, 389-413.

Gonzalo y Tarín, J. 1881. Reseña física y geológica de la Provincia de Granada. Boletín de la Comisión del Mapa Geológico de España, 8, 1-130.

Gonzalo y Tarín, J. 1882a. Edad geológica de las calizas metalíferas de la Sierra de Gádor en la provincia de Almería. Boletín Comisión del Mapa Geológico de España, IX, 97-111. 
Gonzalo y Tarín, J. 1882b. Hallazgo de fósiles triásicos marinos en la Sierra de Gádor, Almería. Anales de la Sociedad de Historia Natural, 11, 37-39.

Gonzalo y Tarín, J. 1885. Dos palabras acerca de la geología de Huelva. Boletín de la Comisión del Mapa Geológico de España, 12, 609-616.

Gonzalo y Tarín, J. 1886-1887. Descripción física, geológica y minera de la provincia de Huelva. Memorias de la Comisión del Mapa Geológico de España, 14, parte I (1886: Descripción física): 1- 274; parte II (1887: Descripción geológica: estratigrafía), 1-671.

Gonzalo y Tarín, J. 1888. Descripción física, geológica y minera de la provincia de Huelva. Parte III: Descripción minera. Memorias de la Comisión del Mapa Geológico de España, 15, 1-660.

Lozano, R.P. y Rábano, I. 2001. Las colecciones históricas de rocas de Barcelona del Museo Geominero (IGME, Madrid): catalogación e interpretación histórica. Boletín Geológico y Minero, 112 (2), 133-146.

Lozano, R.P. y Rábano, I. 2004. Revisión y catalogación de las colecciones históricas de rocas de Zaragoza del Museo Geominero (IGME, Madrid). Boletín Geológico y Minero, 115 (1), 85-102.

Lozano, R.P., Rodrigo, A. y Rábano, I. 1999. Fósiles en las colecciones históricas de rocas del Museo Geominero. II. Aragón. In: Rábano, I. (ed.), Actas de las XV Jornadas de Paleontología y Simposios de los Proyectos PICG 393, 410 y 421. Temas Geológico-Mineros ITGE, 26, 404-408.

Lozano, R.P., Menéndez, S. y Rábano, I. 2005a. La colección Schulz de rocas de Galicia conservada en el Museo Geominero (Instituto Geológico y Minero de España, Madrid). In: Rábano, I. andTruyols, J (eds.), Miscelánea Guillermo Schulz. Cuadernos del Museo Geominero, 5, 191-206.

Lozano, R.P., Rodrigo, A., Menéndez, S. y de la Fuente, M. 2005b. Catálogo de la colección histórica de fósiles de la provincia de Barcelona conservada en el Museo Geominero (Instituto Geológico y Minero de España). Boletín Geológico y Minero, 116 (3), 257-272.

Martínez, G. y Rábano, I. 1999. La colección de nautiloideos jurásicos del Museo Geominero (ITGE, Madrid). In: Rábano, I. (ed.), Actas de las XV Jornadas de Paleontología y Simposios de los Proyectos PICG 393, 410 y 421. Temas Geológico-Mineros ITGE, 26, 415-423.

Menéndez, S. y Quiralte, M.V. 2019. Fósiles históricos de la provincia de Granada en las colecciones paleontológicas del Museo Geominero (IGME, Madrid, España). XXXV
Jornadas de la Sociedad Española de Paleontología, Baza, 187-191.

Menéndez, S. y Rábano, I. 2010. Fósiles de Extremadura en la colección paleontológica histórica del Museo Geominero (Instituto Geológico y Minero de España, Madrid): catálogo y puesta en valor. Boletín Geológico y Minero, 121 (2), 169-178.

Menéndez, S., Rábano, I. y Corrales, B. 2016. Colecciones paleontológicas históricas de la provincia de Huelva conservadas en el Museo Geominero (Instituto Geológico y Minero de España, Madrid). Geo-Temas, 16 (2), 239-241.

Rábano, I. 1998. La colección paleontológica de Casiano de Prado conservada en el Museo Geominero (ITGE, Madrid). Geogaceta, 23, 123-125.

Rábano, I. 1999. Colecciones paleontológicas cordobesas en el Museo Geominero (ITGE, Madrid). Revista de la Asociación Cordobesa de Mineralogía y Paleontología, 37, 17-19.

Rábano, I. 2000. Colecciones históricas de fósiles de la provincia de Jaén en los fondos del Museo Geominero (IGME, Madrid). In: Rábano, I. (ed.), Patrimonio Geológico y Minero en el Marco del Desarrollo Sostenible. Temas Geológico-Mineros IGME, 31, 529- 535.

Rábano, I. 2006. Patrimonio geológico mueble del Instituto Geológico y Minero de España: colecciones paleontológicas históricas del Paleozoico Inferior de la provincia de León en el Museo Geominero. De Re Metallica, 6-7, 7-12.

Rábano, I. 2015. Los Cimientos de la Geología. La Comisión del Mapa Geológico de España (1849-1910). Instituto Geológico y Minero de España, Madrid, 329 pp.

Rábano, I. y Delvene, G. 2003. Colecciones paleontológicas históricas de Aragón, procedentes de la Comisión del Mapa Geológico de España, en el Museo Geominero (Madrid). Naturaleza Aragonesa, 10, 14-24.

Rábano, I. y Gutiérrez-Marco, J.C. 1999. La "Sinopsis" paleontológica de Lucas Mallada: fechas de publicación y otros aspectos editoriales. In: Rábano, R. (ed.), Actas de las XV Jornadas de Paleontología y Simposios de los Proyectos PICG 393, 410 y 421. Temas Geológico-Mineros ITGE, 26, 103-110.

Romero, E. 2013. Escuela de Minas. 11/3/2016,huelvabuenasnoticias.com/2013/04/30/escuela-deminas/

Suárez Suárez, J., Puche Riart, O. y Delgado Domínguez, A. 2017. Gonzalo y Tarín y la Descripción Física, Geológica y Minera de la Provincia de Huelva 1886-1888. Boletín Geológico y Minero, 128 (1), 111-128.

Recibido: julio 2019

Revisado: noviembre 2019

Aceptado: enero 2020

Publicado: marzo 2021 